\title{
Search for inelastic dark matter with the CDMS experiment
}

\author{
Sebastian Arrenberg* \\ for the CDMS Collaboration \\ Physics Institute, University of Zürich \\ E-mail: arrenberg@physik.uzh.ch
}

\begin{abstract}
The Cryogenic Dark Matter Search experiment (CDMS II) employs low-temperature germanium and silicon semiconductors to search for Weakly Interacting Massive Particles (WIMPs) via their elastic scattering off the target nuclei. Results from a reanalysis of the entire five-tower data set with an exposure of $969 \mathrm{~kg}$-days are presented. The analysis window was extended to a recoil energy of $150 \mathrm{keV}$, and an improved surface-event background-rejection cut was defined to increase the sensitivity of the experiment to the inelastic dark matter (IDM) model. Three WIMP candidates were found between $25 \mathrm{keV}$ and $150 \mathrm{keV}$. The probability to observe three or more surface-background events in this energy range is $9 \%$. Due to the occurrence of these events the constraints on the IDM parameter space are slightly less stringent than from our previous analysis with a narrower energy window of $10-100 \mathrm{keV}$.
\end{abstract}

Identification of Dark Matter 2010

July 26 - 302010

University of Montpellier 2, Montpellier, France

* Speaker. 


\section{DAMA/LIBRA and inelastic dark matter}

Cosmological observations strongly suggest that the majority of the matter in the universe consists of non-luminous, non-baryonic matter [1]. This dark matter should be distributed in dark halos of galaxies such as the Milky Way, enabling the direct detection of the dark matter particles via their interactions in terrestrial detectors [2]. The change in the relative velocity of the dark matter particles and the earthbound target due to the movement of the Earth around the Sun is expected to provide an annual modulation of the counting rate [3]. Such a modulation, consistent with the expected signature of galactic dark matter particles interacting in a terrestrial detector, was observed by the DAMA collaboration $[4,5]$ in the electron-equivalent recoil-energy range of 2-6 keV with a periodicity of $0.999 \pm 0.002$ years and a phase of $146 \pm 7$ days [6]. However, an interpretation of this result as a signal from Weakly Interacting Massive Particles (WIMPs) [7] scattering elastically off nuclei is inconsistent with other experimental results $[8,9,10,11,12,13]$.

Inelastic dark matter (IDM) scattering was proposed to overcome this inconsistency [14]. The inelastic scenario assumes that WIMPs can only scatter off baryonic matter by a transition into an excited WIMP state at a certain energy above the ground state, while elastic scattering is forbidden or highly suppressed. The minimal velocity required to produce a recoil of energy $E_{R}$ in such an inelastic scatter is given by

$$
v_{\min }=\frac{1}{\sqrt{2 m_{N} E_{R}}}\left(\frac{m_{N} E_{R}}{\mu}+\delta\right),
$$

where $m_{N}$ is the mass of the target nucleus, $\mu$ is the reduced mass of the WIMP-nucleus system, and $\delta$ is the WIMP-mass splitting; $\delta=0 \mathrm{keV}$ is equivalent to elastic scattering. Important consequences of this model for direct detection experiments are differential rates which peak at tens of keV recoil energy, and a significant suppression for low recoil energies. In addition, the annual modulation signature is significantly enhanced. Finally, it is important to note that the IDM model is particularly sensitive to heavy target nuclei, so rather to xenon and iodine than germanium, and to the escape velocity cutoff of the WIMP-velocity distribution due to the larger minimal velocity $v_{\min }$.

\section{The CDMS experiment}

The Cryogenic Dark Matter Search experiment (CDMS II) operated a total of 19 germanium and 11 silicon crystal detectors, each having a mass of $\sim 230 \mathrm{~g}$ and $\sim 105 \mathrm{~g}$, respectively, at a temperature of $\sim 40 \mathrm{mK}$ in the Soudan Underground Laboratory [15]. These semiconductors were stacked into five towers (T1-T5) with six detectors (Z1-Z6) each. They were instrumented with four channels composed of superconducting transition-edge sensors on the top side to detect the signal from phonon excitation, and two concentric electrodes on the bottom side to measure the ionization signal from each particle interaction simultaneously. The recoil energy was reconstructed from the phonon and the ionization signal. The ratio of ionization to recoil energy, the ionization yield, was used to discriminate nuclear- from electron-recoil events to a very high accuracy. WIMP interactions were expected to be nuclear recoils for this analysis. The main source of misidentified electron recoils were surface events with interactions in the first few $\mu \mathrm{m}$ of the detectors' surfaces. Due to incomplete charge collection these events had a reduced ionization yield and could populate 
the nuclear-recoil acceptance region. Hence, they could mimic WIMP-nucleon interactions. The phonon signals of these surface electron-recoil events had faster-rising pulses than bulk nuclear recoils and occurred closer in time to the nearly prompt ionization pulses. Thus, a cut based on the timing parameters of the events was employed to reject interactions at the detectors' surfaces. These events constituted the dominant background for the CDMS II experiment while the neutron background from cosmogenics and radioactive processes was almost negligible.

\section{Extending the CDMS analysis range}

Initial constraints from CDMS on the inelastic dark matter model interpretation of the DAMA claim had been set using an energy range of $10-100 \mathrm{keV}$ recoil energy [8]. Here a dedicated IDM analysis of the entire CDMS II five-tower data set, taken during two periods of stable operation between October 2006 and July 2007 [16], and four periods between July 2007 and September 2008 [8], is presented. There are two main reasons for performing this reanalysis. From the results of the previous analysis shown in Fig. 3 of [8] it can be observed that the remaining allowed parameter space of the IDM model lies around WIMP masses of $100 \mathrm{GeV} / \mathrm{c}^{2}$ and mass splittings of $120 \mathrm{keV}$. Theoretical predictions employing parameters in that region for the differential recoil spectra in a germanium target can be found in the left panel of Fig. 1. There is a significant contribution to the rates above the previous upper analysis limit of $100 \mathrm{keV}$ so that a simple extension to $150 \mathrm{keV}$ increases the sensitivity. Moreover, the rate drops to zero for low recoil energies, which is different from the elastic scattering case. Since most of the dominant surface-event background occurs at energies just above the $10 \mathrm{keV}$ threshold of previous analyses [16], where no signal is expected, the sensitivity can be further improved by redefining a looser surface-event rejection cut neglecting low recoil energies. The threshold for setting this cut was set to $25 \mathrm{keV}$.

After quality cuts ensuring detector stability and removing periods of poor detector performance, e.g. due to insufficient neutralization, which were taken from the previous analyses $[8,16]$ a total germanium exposure of $969 \mathrm{~kg}$-days was considered for this reanalysis. The silicon detectors were neglected due to their lower sensitivity to inelastic scattering. Given that both data sets had already been analyzed, this was not a blind analysis. However, the analysis was performed in a similar manner such that selection criteria and background estimates were defined and evaluated only on WIMP-search data outside of the signal region and calibration data.

In addition to the quality cuts, most of the cuts defining selection criteria for WIMP-nucleon interactions remained unchanged with respect to the previous analyses $[8,16]$. This included the definition of the single-scatter cut, requiring a signal in any of the other 29 detectors to not exceed the phonon-noise level, the fiducial-volume cut, rejecting events at the edges of the detectors, and the muon-veto cut, demanding no coincident energy to be deposited in the active muon veto surrounding the apparatus.

Extending the analysis window to $150 \mathrm{keV}$ was impeded by the fact that statistics from the californium neutron source were low above $\sim 100 \mathrm{keV}$. This source was used to calibrate the detectors, and, in particular, to define the WIMP-acceptance region, a $2 \sigma$ nuclear-recoil band in the ionization-yield versus recoil-energy plane. Thus, to a certain extent, extrapolations were necessary to define the nuclear-recoil bands at higher energies. Their validity was examined by combining statistics from all six runs for each detector and comparisons with theoretical predictions from 

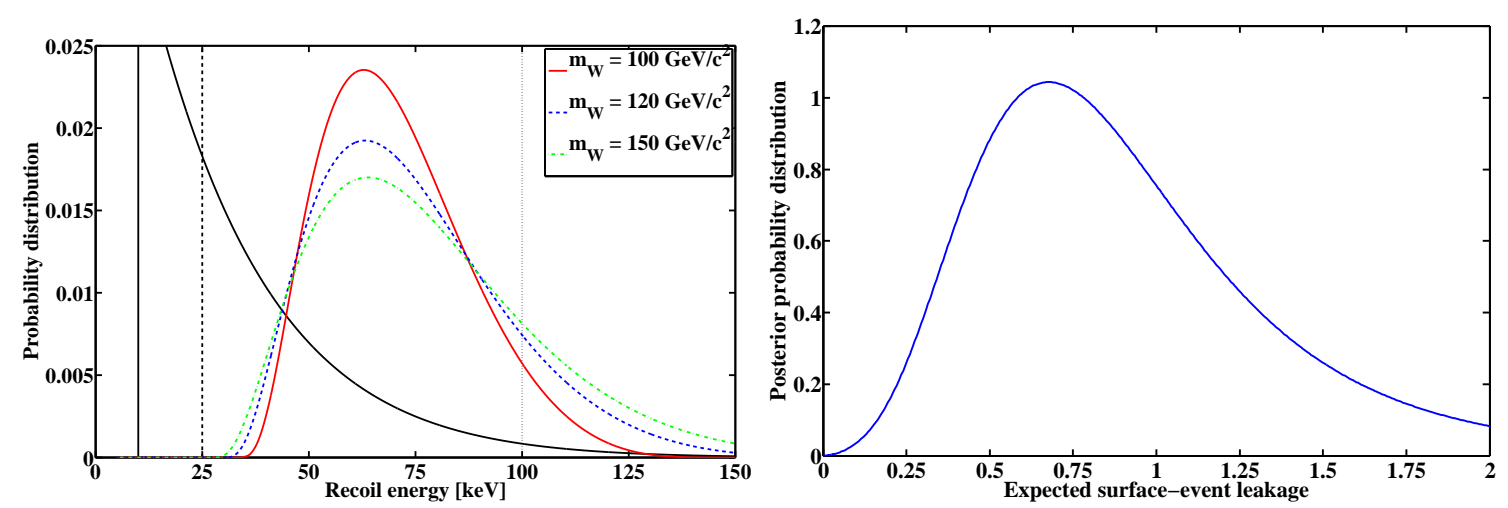

Figure 1: Left panel: Differential recoil spectra in a germanium target for a WIMP-mass splitting $\delta$ of $120 \mathrm{keV}$ and a few representative WIMP masses $m_{W}$. For comparison the spectrum for a WIMP with a mass of $120 \mathrm{GeV} / \mathrm{c}^{2}$ assuming elastic scattering $(\delta=0 \mathrm{keV})$ is also shown (black/solid). The spectra are normalized to unity in the $5-150 \mathrm{keV}$ recoil-energy range. Right panel: Distribution of the surface-event leakage estimate in the $25-150 \mathrm{keV}$ energy range. See text for details.

Lindhard theory $[17,18]$ which showed a good agreement. The fact that the bands as well as the corresponding efficiencies of the nuclear-recoil cut had only a minor energy dependence above $\sim 25 \mathrm{keV}$ assured confidence that these extrapolations were justified.

Surface-event rejection was based on the sum of the rise time of the largest phonon pulse and its delay relative to the ionization pulse. This timing cut was set in the $25-150 \mathrm{keV}$ energy range using only barium and californium calibration data. The cut was tuned to yield a maximum spectrum averaged exposure for WIMP masses of $100 \mathrm{GeV} / \mathrm{c}^{2}$ and mass splittings of $120 \mathrm{keV}$ given a fixed leakage of 0.6 events for the whole data set. This leakage was determined to give the best sensitivity by a Monte Carlo simulation.

Since the timing cut was set using calibration data and it was know from previous analyses that there are differences to the WIMP-search data, e.g. considering the recoil-energy and ionizationyield distributions [8], a more refined leakage calculation was accomplished. The leakage was estimated by multiplying the number of WIMP-search nuclear-recoil single scatters failing the timing cut by pass-fail ratios deduced from event samples which were assumed to resemble the population of background events. Two classes of events were used to independently estimate the ratios and expected background: WIMP-search multiple scatters within the nuclear-recoil band and surrounding the nuclear-recoil band. Due to the low number of events passing the timing cut a dedicated Bayesian surface-event leakage estimate was applied [19]. The final background distribution obtained by combining the estimates from both pass-fail ratios is shown in the right panel of Fig. 1. It contains all statistical and systematic errors. It has a maximum around 0.6 events where the leakage had been fixed for the setting of the cut while the median, which was used as the final background estimate, is slightly higher but agrees with this value within error bars:

$$
\mu_{25-150 \mathrm{keV}}=0.8_{-0.3}^{+0.5}(\text { stat. })_{-0.2}^{+0.3} \text { (syst.) } \text {. }
$$

The neutron background from cosmogenics and radioactive processes is still under investigation but is expected to be negligible. 
The final efficiency from this reanalysis including all cuts, based on the entire CDMS II fivetower data set, was larger than the efficiency from the previous WIMP analysis, based only on the last four data runs and the $10-100 \mathrm{keV}$ energy range [8], by a factor of $\sim 1.5$. This was due to the fact that the low-energy range, where most of the background occurred but no signal was expected in the IDM model, was neglected while redefining the timing cut.

After "unblinding", three events were observed in the acceptance region within the $25-150 \mathrm{keV}$ recoil-energy range passing the surface-event rejection cut. It was verified that the performance of the experiment was stable at the times at which the events occurred. One WIMP candidate was observed at $37.3 \mathrm{keV}$ in detector T4Z6. This was a detector located at the bottom of its tower with no adjacent detector below, making it particularly difficult to reject background events by detecting multiple scatters. A second event occurred at $73.3 \mathrm{keV}$ in T4Z2 very close above the timing-cut boundary. Finally, a candidate was observed in T1Z2 at $129.5 \mathrm{keV}$ above the upper analysis range from previous analyses. This event was far above the cut boundary and would be rejected neither by the surface-event cut from the previous analysis [8], nor a refined timing cut tuned to a fixed leakage of 0.1 , instead of 0.6 , events.

The probability to observe three or more surface-leakage events given the background distribution shown in the right panel of Fig. 1 is $9 \%$ which is low but not negligible. Thus, a significant evidence for a WIMP detection cannot be claimed, while non of the candidates can be rejected as background.

\section{Constraining the IDM parameter space}

Spin-independent cross-section upper limits at 90\% C.L. were computed for given WIMP masses and WIMP-mass splittings using the optimum interval method [20], conservatively treating all WIMP candidates as signal. The differential rates were calculated under standard halo assumptions according to [21]. The escape velocity was assumed to be $544 \mathrm{~km} / \mathrm{s}$ [22] while the standard value of $220 \mathrm{~km} / \mathrm{s}$ was applied for the dispersion of the Maxwellian dark matter velocity distribution. Helm form factors and a three-dimensional parametrization of the Earth velocity were used according to [18].

Regions allowed by DAMA/LIBRA at four different C.L.s (90, 95, 99, 99.9\%) were computed based on the published modulated spectrum in [6] from an exposure of 1.17 ton-years. As in [8] we followed the $\chi^{2}$ goodness-of-fit technique advocated in [21] to investigate the compatibility between the results from DAMA/LIBRA and CDMS. Quenching factors of 0.30 and 0.09 were applied for sodium and iodine nuclei in the DAMA/LIBRA setup, respectively [23]. Possible channeling effects [24] were not included in this study since they do not have a significant impact on the results from this analysis [25].

Selected results from these computations are shown in Fig. 2 in the cross-section versus WIMP-mass plane for WIMP-mass splittings of $90 \mathrm{keV}$ and $130 \mathrm{keV}$. Apart from the regions allowed by DAMA/LIBRA, and constraints emerging from the analysis presented here, the plots also contain cross-section limits from our previous analysis of the $10-100 \mathrm{keV}$ energy range [8]. Constraints from the new analysis are less stringent due to the occurrence of the three candidates at energies where the rate is expected to peak for higher WIMP-mass splittings. WIMP masses above $60 \mathrm{GeV} / \mathrm{c}^{2}$ and $140 \mathrm{GeV} / \mathrm{c}^{2}$ are excluded for mass splittings of $90 \mathrm{keV}$ and $130 \mathrm{keV}$, respectively, 

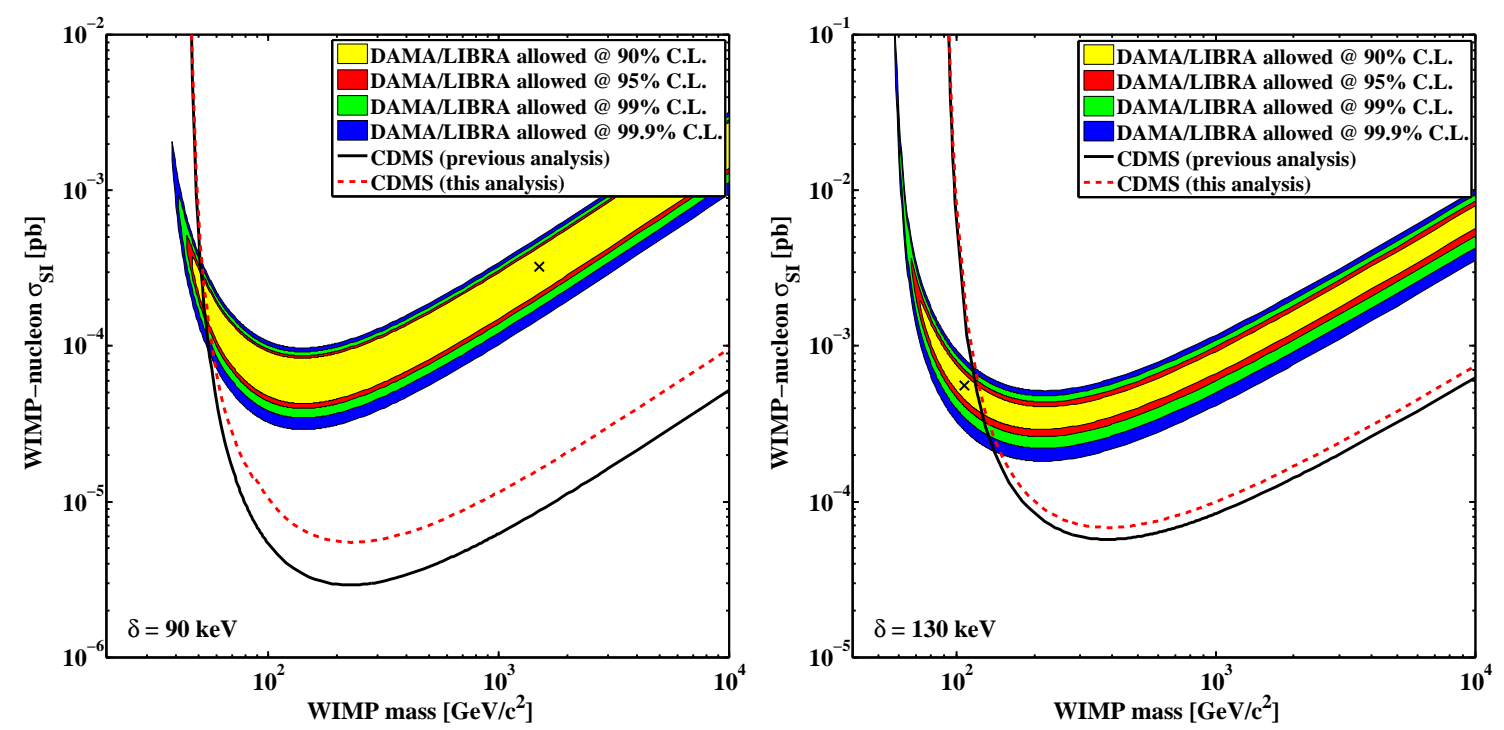

Figure 2: $90 \%$ C.L. upper limits on the scalar WIMP-nucleon cross section for WIMP-mass splittings of $90 \mathrm{keV}$ (left panel) and $130 \mathrm{keV}$ (right panel) from this analysis (red/dashed) and from our previous analysis [8] (black/solid). The colored areas represent DAMA/LIBRA allowed regions at four different C.L.s (90, 95, 99, 99.9\%). The cross $(\times)$ marks the parameter point which yields the minimum $\chi^{2}$ in the shown cross-section versus WIMP-mass plane for the given fixed WIMP-mass splitting.

by the current and previous analysis. An investigation of the full three-dimensional IDM parameter space, consisting of the cross section, WIMP mass, and WIMP-mass splitting is relegated to a subsequent publication [26].

\section{Summary}

The first CDMS analysis which includes recoil energies up to $150 \mathrm{keV}$ was presented. All six data runs with five towers were used in a combined analysis. Due to the occurrence of three candidate events the constraints on the IDM parameter space are weaker than from our previous analysis where no events were observed at intermediate energies, where the rate is expected to peak. Even though this analysis was performed with regard to the IDM scenario the expansion of the analysis range to $150 \mathrm{keV}$ could be useful to test other models predicting a signal at tens of $\mathrm{keV}$ recoil energy.

\section{References}

[1] E. Komatsu et al. (WMAP), Astrophys. J. Suppl. 180, 330 (2009).

[2] M. W. Goodman and E. Witten, Phys. Rev. D 31, 3059 (1985).

[3] A. K. Drukier, K. Freese and D. N. Spergel, Phys. Rev. D 33, 3495 (1986).

[4] R. Bernabei et al. (DAMA), Phys. Lett. B 480, 23 (2000).

[5] R. Bernabei et al. (DAMA), Eur. Phys. J. C 56, 333 (2008).

[6] R. Bernabei et al. (DAMA), Eur. Phys. J. C 67, 39 (2010). 
[7] G. Bertone, D. Hooper and J. Silk, Phys. Rept. 405, 279 (2005).

[8] Z. Ahmed et al. (CDMS II), Science 327, 1619 (2010).

[9] E. Aprile et al. (XENON100), Phys. Rev. Lett. 105, 131302 (2010).

[10] J. Angle et al. (XENON10), Phys. Rev. Lett. 101, 091301 (2008).

[11] E. Behnke et al. (COUPP), Science 319, 933 (2008).

[12] H. S. Lee et al. (KIMS), Phys. Rev. Lett. 99, 091301 (2007).

[13] V. N. Lebedenko et al. (ZEPLIN), Phys. Rev. D 80, 052010 (2009).

[14] D. Smith and N. Weiner, Phys. Rev. D 64, 043502 (2001).

[15] D. S. Akerib et al., Phys. Rev. D 72, 052009 (2005).

[16] Z. Ahmed et al. (CDMS II), Phys. Rev. Lett. 102, 011301 (2009).

[17] J. Lindhard et al., K. Dan. Vidensk. Selsk., Mat.-Fys. Medd. 33, 10 (1963).

[18] J. D. Lewin and P. F. Smith, Astropart. Phys. 6, 87 (1996).

[19] J. P. Filippini, Ph.D. thesis, University of California Berkeley (2008).

[20] S. Yellin, Phys. Rev. D 66, 032005 (2002).

[21] C. Savage, G. Gelmini, P. Gondolo and K. Freese, JCAP 04, 010 (2009).

[22] M. C. Smith et al., Mon. Not. Roy. Astron. Soc. 379, 755 (2007).

[23] R. Bernabei et al. (DAMA), Phys. Lett. B 389, 757 (1996).

[24] R. Bernabei et al. (DAMA), Eur. Phys. J. C 53, 205 (2008).

[25] N. Bozorgnia, G. B. Gelmini and P. Gondolo, JCAP 11, 019 (2011); JCAP 11, 028 (2010).

[26] Z. Ahmed et al. (CDMS II), to be published. 